\title{
Predicting Polysomnography Parameters from Anthropometric Features and Breathing Sounds Recorded during Wakefulness
}

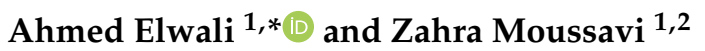 \\ 1 Biomedical Engineering Graduate Program, University of Manitoba, Winnipeg, MB R3T 5V6, Canada; \\ Zahra.Moussavi@umanitoba.ca \\ 2 Electrical and Computer Engineering Department, University of Manitoba, Winnipeg, MB R3T 5V6, Canada \\ * Correspondence: Elwalia@myumanitoba.ca
}

Citation: Elwali, A.; Moussavi, Z. Predicting Polysomnography Parameters from Anthropometric Features and Breathing Sounds Recorded during Wakefulness. Diagnostics 2021, 11, 905. https://doi.org/10.3390/ diagnostics11050905

Academic Editor: Bilgay Izci Balserak

Received: 11 February 2021

Accepted: 14 May 2021

Published: 19 May 2021

Publisher's Note: MDPI stays neutral with regard to jurisdictional claims in published maps and institutional affiliations.

Copyright: (C) 2021 by the authors. Licensee MDPI, Basel, Switzerland. This article is an open access article distributed under the terms and conditions of the Creative Commons Attribution (CC BY) license (https:// creativecommons.org/licenses/by/ $4.0 /)$.

\begin{abstract}
Background: The apnea/hypopnea index (AHI) is the primary outcome of a polysomnography assessment (PSG) for determining obstructive sleep apnea (OSA) severity. However, other OSA severity parameters (i.e., total arousal index, mean oxygen saturation ( $\mathrm{SpO} 2 \%$ ), etc.) are crucial for a full diagnosis of OSA and deciding on a treatment option. PSG assessments and home sleep tests measure these parameters, but there is no screening tool to estimate or predict the OSA severity parameters other than the AHI. In this study, we investigated whether a combination of breathing sounds recorded during wakefulness and anthropometric features could be predictive of PSG parameters. Methods: Anthropometric information and five tracheal breathing sound cycles were recorded during wakefulness from 145 individuals referred to an overnight PSG study. The dataset was divided into training, validation, and blind testing datasets. Spectral and bispectral features of the sounds were evaluated to run correlation and classification analyses with the PSG parameters collected from the PSG sleep reports. Results: Many sound and anthropometric features had significant correlations (up to 0.56 ) with PSG parameters. Using combinations of sound and anthropometric features in a bilinear model for each PSG parameter resulted in correlation coefficients up to 0.84 . Using the evaluated models for classification with a two-class random-forest classifier resulted in a blind testing classification accuracy up to $88.8 \%$ for predicting the key PSG parameters such as arousal index. Conclusions: These results add new value to the current OSA screening tools and provide a new promising possibility for predicting PSG parameters using only a few seconds of breathing sounds recorded during wakefulness without conducting an overnight PSG study.
\end{abstract}

Keywords: obstructive sleep apnea; screening; machine learning; correlation; trachea; sleep report

\section{Introduction}

Obstructive sleep apnea (OSA) is a common disorder characterized by repetitive partial or complete episodes of airflow obstruction that can result in intermittent hypoxia, transient hypercapnia, and arousals from sleep. Commonly, the severity of OSA is mainly determined by the apnea/hypopnea index (AHI), which represents the number of apnea/hypopnea events per hour of sleep. Based on the AHI values for adults, the severity of OSA is categorized into no-OSA (AHI $<5)$, mild $(5 \leq \mathrm{AHI}<15)$, moderate $(15 \leq \mathrm{AHI}<30$ ), and severe OSA $(\mathrm{AHI} \geq 30)$ [1]. However, many other parameters are measured during overnight polysomnography (PSG), the gold standard of sleep apnea detection.

Approximately 1 billion of the world's population between the ages of 30 and 69 years are estimated to have OSA [2]; yet, it is still underdiagnosed [3]. OSA diagnosis and recommending a treatment option using the gold-standard PSG are expensive, laborious, and time-consuming; also, PSG is not available in remote areas. There are portable PSG monitoring devices that can be lent to individuals to assess their OSA overnight at home. However, to recommend a treatment option, there is still a need for a full PSG study at a sleep center. There are questionnaires such as STOP-BANG or the Epworth sleepiness 
score for a quick OSA screening, but they lack objectivity and have very poor specificity $(\sim 20)[4,5]$, which can increase the waiting list and lead to unnecessary referrals to sleep centers for full PSG studies; hence, adding unnecessary costs to the health care system. On the other hand, undiagnosed OSA, in particular, increases the perioperative morbidity and mortality risks for OSA patients undergoing surgery requiring full anesthesia [6]. Therefore, there is a need for a quick, objective screening tool to aid fast and accurate results with high sensitivity and specificity of OSA detection.

Our previous studies $[7,8]$ have shown the ability to predict the severity of OSA, based on AHI, by analyzing a few minutes of breathing sounds recorded during wakefulness. We also demonstrated the effects of anthropometric information on the classification process [7,9]. Our latest OSA screening algorithm, called AWakeOSA [8], resulted in a blind testing accuracy $>81 \%$ with a balanced specificity and sensitivity with a threshold of $\mathrm{AHI} \geq 15$. The threshold of AHI $\geq 15$ was used because it is the most clinically accepted threshold to identify individuals with OSA who might benefit from the treatment [10].

Currently, AHI is the primary PSG parameter for determining an individual's OSA severity. However, other PSG parameters, such as total time of sleep (TST), total arousal index, and mean oxygen saturation (SpO2\%) of TST, are also important for a full clinical diagnosis of OSA [11]. Using the AHI parameter alone neglects events' duration, oxygen desaturation depth, the difference of impact between apneic and hypopnic events, etc. Nevertheless, knowing these parameters helps with understanding one's OSA pathology and choosing the proper treatment [12].

The upper airway (UA) of OSA patients is characterized by a structural deformation, narrower cross-sectional area, and more regional stiffness compared to those of healthy individuals $[13,14]$. These changes of the UA affect breathing sounds as they are generated by the flow of air in the UA [15]. To the best of our knowledge, there is no published work predicting PSG parameters, other than AHI, using a screening tool conducted during wakefulness. Therefore, this study for the first time demonstrates the proof of concept of a technique to predict PSG parameters representing OSA severity without a sleep study and only using a few tracheal breathing sounds recorded during wakefulness and the person's anthropometric information such as neck circumference (NC), body mass index (BMI), etc. Hence, we hypothesize that breathing sounds characteristics (features) would be representative of the OSA severity. More specifically, we hypothesize that tracheal breathing sounds have features capable of predicting PSG parameters such as total arousal index, mean $\mathrm{SpO} 2 \%$, etc. that are related to OSA severity.

\section{Materials and Methods}

We attempted to solve a two-class classification problem to predict each PSG parameter separately. The data of each PSG parameter was divided into two severity classes/groups based on a threshold (see Section 2.3). One of the two groups represented the normal case, while the other represented the abnormal case. For each PSG parameter, a classification threshold was evaluated, distinctive features were selected, and a model was created using anthropomorphic parameters and breathing sound features. Then the model was used in a classification approach to predict the PSG parameter's severity group (normal or abnormal); violating the threshold (in the abnormal group) implied a high risk of OSA severity; check Figure 1 for the general flow of the process.

\subsection{Study Population}

The data for this study were adopted from our previous study [8]. During wakefulness, about 1-2 h prior to conducting the PSG study at Sleep Disorders Center in Misericordia Health Centre (Winnipeg, Canada), breathing sound recording was performed. Patients diagnosed with any other respiratory diseases were excluded. We obtained the PSG study report of the participants after a sleep technician completed their overnight PSG assessment analysis. All study participants signed an informed consent approved by the Biomedical Research Ethics Board of the University of Manitoba (approved on 27 October 2011) prior 
to the breathing experiment. Out of the 199 individuals in our previous study, data of the 145 (60 females) participants who had a complete PSG report were considered in this study.

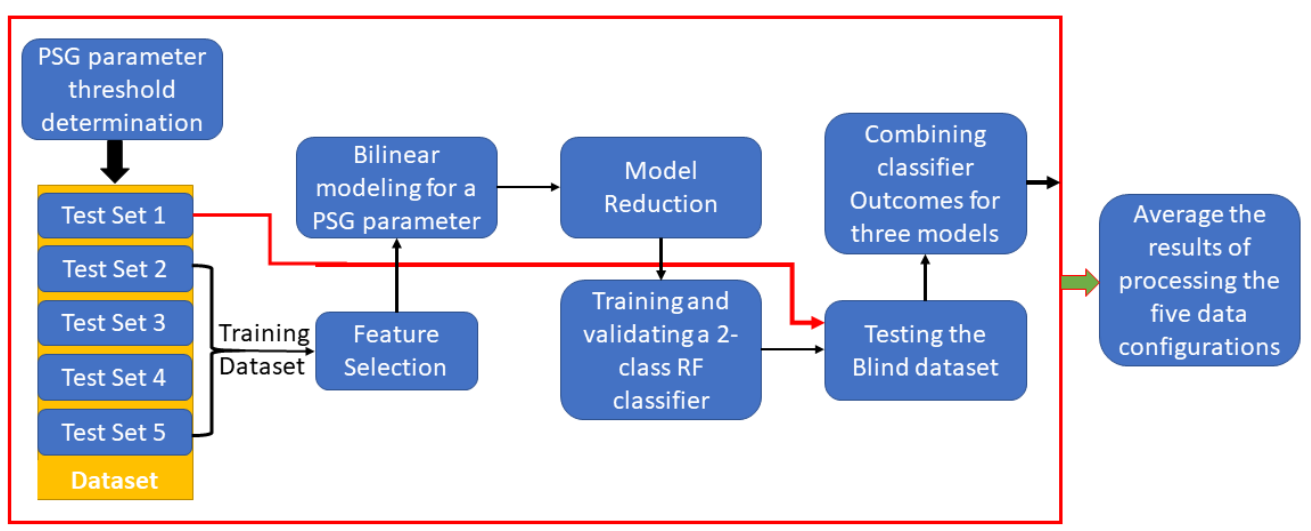

Figure 1. The general flow chart of the process.

\subsection{Procedure and Measurements}

Prior to recording, we collected the height, weight, age, Mallampati score (MpS), neck circumference, and smoking history of each participant. For simplification, the AHI of 15 was used to represent the anthropometric parameters in the two severity groups. We checked whether the data followed the normal distribution for each anthropometric parameter by using the Lilliefors test. The two severity groups were only matched in terms of age, and a $t$-test was used.

All participants conducted a full overnight PSG study. The Sleep Centre used the Natus Sandman PSG collection system (Natus Canada, Oakville, ON, Canada), which recorded 6 channels of electroencephalogram signals, chin and leg electromyography signals, electrooculography signals, electrocardiogram signals, snoring sounds, thorax and abdomen efforts signals, air flow and pressure signals, pulse and $\mathrm{SpO} 2$ signals, transcutaneous oxygen pressure signals, and video image for body position detection. A full overnight PSG study reports 79 different parameters reflecting on one's OSA severity; they are listed in Table A1 of the Appendix A. The data in these reports were used for verification of our predictive modeling outcomes.

Tracheal Breathing Sounds

The tracheal breathing sounds were recorded using a Sony microphone (ECM77BAdvance Electronics, Winnipeg, MB, Canada) during wakefulness in a supine position. The microphone was placed over the suprasternal notch of the trachea using a double-sided adhesive ring tape. The acoustic signals were amplified, band-pass filtered $(0.05-5000 \mathrm{~Hz}$, Biopac DA100C - BIOPAC Systems Inc., Goleta, CA, USA), and sampled at 10,240 Hz. Participants were instructed to breathe five deep breaths through their nose, then another five cycles through their mouth; for more details on the recording protocol and the preprocessing stage, see [15]. The spectra and bi-spectra of the signals were estimated using the welsh method [16] and an indirect class conventional bispectrum estimator [17], respectively. The breathing sounds' characteristic features, used to predict AHI in our previous study [8], were also used to predict the PSG parameters (other than AHI) in this study (see Table 1 for the list of features). 
Table 1. Descriptions and details of the selected anthropometric and sound features.

\begin{tabular}{|c|c|c|}
\hline FL & BM & Feature's Definition \\
\hline $\mathrm{F} 1$ & - & Body mass index (BMI) \\
\hline $\mathrm{F} 2$ & - & Age \\
\hline F3 & - & Sex \\
\hline $\mathrm{F} 4$ & - & Weight \\
\hline F5 & - & Neck circumference (NC) \\
\hline F6 & - & Mallampati score (MpS) \\
\hline F7 & InsN & $\begin{array}{l}f 2=525 \\
f 1=350\end{array}$ Mean of $P(f)$ \\
\hline F8 & ExpM & $\begin{array}{l}f 2=130 \\
f 1=235\end{array}$ Mean of $P(f)-{ }_{f 1=1260}^{f 2=1410}$ Mean of $P(f)$ \\
\hline F9 & InsN & $\begin{array}{l}f 2=115 \\
f 1=275\end{array}$ Mean of $P(f)-{ }_{f 1=915}^{f 2=1240}$ Mean of $P(f)$ \\
\hline F10 & InsM & $\begin{array}{l}f 2=1800 \\
f 1=75\end{array} \quad$ Frequency of the first peak of $P(f)$ \\
\hline F11 & InsM & $\begin{array}{l}f 2=1700 \\
f 1=75\end{array}$ Bandwidth of the spectral centroid of $P(f)$ \\
\hline F12 & ExpM & $\begin{array}{l}f 2=550 \\
f 1=300\end{array}$ Bandwidth of the spectral centroid of $P(f)$ \\
\hline F13 & InsM & $\begin{array}{l}f 2=600 \\
f 1=0\end{array} \quad$ Frequency of the first peak of $P(f)$ using zero-crossing \\
\hline F14 & InsM & Total $B(f, f)$ entropy \\
\hline F15 & InsM & $\begin{array}{l}f 2=1515 \\
f 1=1200\end{array}$ First - order moment of the positive diagonal of $B(f, f)$ \\
\hline F16 & InsM & $\begin{array}{l}f 2=1515 \\
f 1=1200\end{array}$ First - order moment of the negative diagonal of $B(f, f)$ \\
\hline F17 & ExpM & $\begin{array}{l}f 2=235 \\
f 1=130\end{array}$ Second - order moment of the $0.5 f-f$ line of $B(f, f)$ \\
\hline F18 & InsN & Weight center of the positive diagonal of $B(f, f)$ \\
\hline F19 & InsN & $\begin{array}{l}f 2=355 \\
f 1=250\end{array}$ Mean of the positive diagonal of $B(f, f)$ \\
\hline $\mathrm{F} 20$ & InsN & $\begin{array}{l}f 2=355 \\
f 1=250\end{array}$ Weight center of $B(f, f)$ \\
\hline $\mathrm{F} 21$ & InsN & $\begin{array}{l}f 2=275 \\
f 1=115\end{array}$ First - order moment of the positive diagonal of $B(f, f)$ \\
\hline $\mathrm{F} 22$ & InsN & $\begin{array}{l}f 2=525 \\
f 1=350\end{array}$ Weight center of the negative diagonal of $B(f, f)$ \\
\hline F23 & InsN & $\begin{array}{l}f 2=350 \\
f 1=245\end{array}$ Mean of the slope of $P(f)$ \\
\hline $\mathrm{F} 24$ & InsN & $\begin{array}{l}f 2=350 \\
f 1=100\end{array}$ Bandwidth of the spectral centroid of $P(f)$ \\
\hline F25 & InsM & Higuchi fractal dimension \\
\hline F26 & InsM & $\begin{array}{l}f 2=270 \\
f 1=140\end{array}$ Geometric Mean of $B(f, f)$ \\
\hline $\mathrm{F} 27$ & InsM & Total Weight center of $B(f, f)$ \\
\hline $\mathrm{F} 28$ & InsM & $\begin{array}{l}f 2=270 \\
f 1=140\end{array}$ First - order moment of the $2 f-f$ line of $B(f, f)$ \\
\hline F29 & ExpM & Total Weight center of $B(f, f)$ \\
\hline F30 & InsN & $\begin{array}{l}f 2=275 \\
f 1=130\end{array}$ Mean of $B(f, f)$ \\
\hline F31 & InsN & $\begin{array}{l}f 2=275 \\
f 1=130\end{array}$ Second - order moment of the positive diagonal of $B(f, f)$ \\
\hline F32 & InsM & $\begin{array}{l}f 2=230 \\
f 1=130\end{array}$ Mean of $P(f)$ \\
\hline F33 & InsN & $\begin{array}{l}f 2=280 \\
f 1=130\end{array}$ Mean of $P(f)$ \\
\hline F34 & InsN & $\begin{array}{l}f 2=340 \\
f 1=240\end{array}$ Mean of the slope of $P(f)$ \\
\hline
\end{tabular}


Table 1. Cont.

\begin{tabular}{|c|c|c|}
\hline FL & $\mathbf{B M}$ & Feature's Definition \\
\hline F35 & InsN & $\begin{aligned} f 2 & =350 \\
f 1 & =80\end{aligned}$ Bandwidth of the spectral centroid of $P(f)$ \\
\hline F36 & InsN & $\begin{array}{l}f 2=560 \\
f 1=80\end{array}$ Spectral centroid of $P(f)$ \\
\hline F37 & InsM & $\begin{array}{l}f 2=1800 \quad \text { Frequency of the first peak of } P(f) \text { using zero-crossing } \\
f 1=0\end{array}$ \\
\hline F38 & InsM & TotalWeight center of the positive of diagonal $B(f, f)$ \\
\hline F39 & InsM & $\begin{array}{l}f 2=230 \\
f 1=130\end{array}$ First - order moment of the $2 f-f$ line of $B(f, f)$ \\
\hline F40 & InsM & $\begin{array}{l}f 2=230 \\
f 1=130\end{array}$ Second - order moment of the $2 f-f$ line of $B(f, f)$ \\
\hline $\mathrm{F} 41$ & InsN & $\begin{aligned} f 2=280 \\
f 1=130\end{aligned}$ Mean of $B(f, f)$ \\
\hline F42 & InsN & $\begin{array}{l}f 2=280 \\
f 1=130\end{array}$ Mean of the negative diagonal of $B(f, f)$ \\
\hline F43 & InsN & $\begin{array}{l}f 2=280 \\
f 1=130\end{array}$ Second - order moment of the negative diagonal of $B(f, f)$ \\
\hline $\mathrm{F} 44$ & ExpM & $\begin{array}{l}f 2=485 \\
f 1=375\end{array}$ Mean of $P(f)$ \\
\hline $\mathrm{F} 45$ & InsN & $\begin{array}{l}f 2=500 \\
f 1=360\end{array}$ Mean of $P(f)$ \\
\hline F46 & InsN & $\begin{array}{l}f 2=1250 \\
f 1=1010\end{array}$ Mean of $P(f)$ \\
\hline $\mathrm{F} 47$ & InsN & $\begin{array}{l}f 2=370 \\
f 1=270\end{array}$ Mean of the slope of $P(f)$ \\
\hline $\mathrm{F} 48$ & $\operatorname{ExpN}$ & $\begin{array}{l}f 2=550 \\
f 1=450\end{array}$ Mean of the slope of $P(f)$ \\
\hline F49 & ExpM & $\begin{array}{l}f 2=600 \\
f 1=200\end{array}$ Bandwidth of the spectral centroid of $P(f)$ \\
\hline F50 & InsN & $\begin{array}{l}f 2=620 \\
f 1=440\end{array}$ Spectral centroid of $P(f)$ \\
\hline F51 & ExpN & $\begin{array}{l}f 2=600 \\
f 1=300\end{array}$ Bandwidth of the spectral centroid of $P(f)$ \\
\hline F52 & InsM & $\begin{array}{l}f 2=510 \\
f 1=390\end{array}$ Weight center of the $2 f-f$ line of $B(f, f)$ \\
\hline F53 & ExpM & $\begin{array}{l}f 2=390 \\
f 1=270\end{array}$ Weight center of the negative diagonal of $B(f, f)$ \\
\hline F54 & ExpM & $\begin{array}{l}f 2=390 \\
f 1=270\end{array}$ Weight center of the positive diagonal of $B(f, f)$ \\
\hline F55 & ExpN & $\begin{array}{l}f 2=550 \\
f 1=450\end{array}$ Mean of the negative diagonal of $B(f, f)$ \\
\hline F56 & InsN & $\begin{array}{l}f 2=510 \\
f 1=355\end{array}$ Mean of $P(f) /_{f 1=820}^{f 2=1070}$ Mean of $P(f)$ \\
\hline F57 & InsN & $\begin{array}{l}f 2=350 \\
f 1=250\end{array}$ Mean of the slope of $P(f)$ \\
\hline F58 & InsM & $\begin{array}{l}f 2=550 \\
f 1=90\end{array}$ \\
\hline F59 & InsN & $\begin{array}{l}f 2=550 \quad \text { Frequency of the first peak of } P(f) \text { using zero-crossing } \\
f 1=0\end{array}$ \\
\hline F60 & InsM & $\begin{array}{l}f 2=250 \\
f 1=120\end{array}$ Mean of $B(f, f)$ \\
\hline F61 & InsM & $\begin{array}{l}f 2=250 \\
f 1=120\end{array}$ First - order moment of the positive diagonal of $B(f, f)$ \\
\hline F62 & InsN & $\begin{array}{l}f 2=510 \\
f 1=355\end{array}$ Weight center of the negative diagonal of $B(f, f)$ \\
\hline F63 & InsN & $\begin{array}{l}f 2=1070 \text { Second }- \text { order moment of the } 2 f-f \text { line of } B(f, f) \\
f 1=820\end{array}$ \\
\hline F64 & ExpN & $\begin{array}{l}f 2=600 \\
f 1=100\end{array}$ Weight center of the $0.5 f-f$ line of $B(f, f)$ \\
\hline F65 & InsN & $\begin{array}{l}f 2=520 \\
f 1=395\end{array}$ Mean of $P(f)$ \\
\hline F66 & InsM & $\begin{array}{l}f 2=600 \\
f 1=60\end{array} \quad$ Frequency of the peak of $P(f)$ \\
\hline
\end{tabular}


Table 1. Cont.

\begin{tabular}{|c|c|c|}
\hline FL & BM & Feature's Definition \\
\hline F67 & InsN & $\begin{array}{l}f 2=550 \\
f 1=150\end{array}$ Spectral centroid of $P(f)$ \\
\hline F68 & InsM & $\begin{array}{l}f 2=600 \\
f 1=60\end{array}$ Weight center of the $0.5 f-f$ line of $B(f, f)$ \\
\hline F69 & InsM & $\begin{array}{c}f 2=600 \\
f 1=60\end{array}$ Second - order moment of the $2 f-f$ line of $B(f, f)$ \\
\hline F70 & InsN & $\begin{array}{l}f 2=520 \\
f 1=395\end{array}$ Weight center of the positive diagonal of $B(f, f)$ \\
\hline F71 & InsN & $\begin{array}{l}f 2=520 \\
f 1=395\end{array}$ Mean of the negative diagonal of $B(f, f)$ \\
\hline F72 & ExpN & $\begin{array}{l}f 2=600 \\
f 1=100\end{array}$ \\
\hline F73 & InsM & $\begin{array}{l}f 2=230 \\
f 1=130\end{array}$ Mean of $P(f) /{ }_{f 1=1260}^{f 2=1460}$ Mean of $P(f)$ \\
\hline F74 & InsM & $\begin{array}{l}f 2=1460 \\
f 1=1090\end{array}$ First - order moment of the positive diagonal of $B(f, f)$ \\
\hline F75 & InsM & $\begin{array}{l}f 2=1460 \\
f 1=1260\end{array}$ First - order moment of the positive diagonal of $B(f, f)$ \\
\hline F76 & InsM & $\begin{array}{l}f 2=1460 \\
f 1=1090\end{array}$ \\
\hline F77 & InsM & $\begin{array}{l}f 2=1460 \\
f 1=1260\end{array}$ First - order moment of the $0.5 f-f$ line of $B(f, f)$ \\
\hline F78 & ExpM & $\begin{array}{l}f 2=1410 \\
f 1=1260\end{array}$ First - order moment of the negative diagonal of $B(f, f)$ \\
\hline F79 & InsN & $\begin{array}{l}f 2=1600 \\
f 1=300\end{array}$ First - order moment of the $0.5 f-f$ line of $B(f, f)$ \\
\hline
\end{tabular}

Ins/Exp: inspiration/expiration, $\mathrm{M} / \mathrm{N}$ : mouth/nose, mean: arithmetic mean, $P(f)$ : the power spectrum, $B(f, f)$ : the bispectrum, $f$ : frequency, FL: feature label, and BM: breathing maneuver.

\subsection{Threshold Determination, Data Preparation, and Feature Selection}

Classification threshold determination for each PSG Parameter-80\% of the data were used in the threshold determination process. In order to find a proper threshold for each PSG parameter, we scaled the PSG parameter's values to be normally distributed with an absolute skewness less than 0.5. Basically, if the skewness was $\leq-0.5$, the PSG parameter was squared, and if the skewness was $\geq 0.5$, the logarithm scale of the PSG parameter was used. Then, the overall range of a PSG parameter's values was divided into 20 equal divisions to form threshold candidates for the classification process. For example, the TST range was between 0.5 and $7 \mathrm{~h}$, and it had a skewness $<0.5$; thus, it did not require scaling. With the above 20 equal divisions, the potential classification thresholds were found as $0.825,1.15,1.475, \ldots 6.675$.

Classification threshold selection-For each threshold candidate of a PSG parameter, the power spectra of the sound signals of those with PSG parameter values less than the candidate threshold were grouped together, and the same was done for those with values higher than the candidate threshold. The average curve for each of these two groups was calculated with its $95 \%$ confidence interval. At some frequency bandwidths, there was no overlap between the curves of the two groups; see Figure 2 for the gaps (i.e., $150-300 \mathrm{~Hz}$, $720-900 \mathrm{~Hz}, 1050-1120 \mathrm{~Hz}$, and 1450-1700 Hz). Then, the average absolute difference between the $95 \%$ confidence intervals' boundaries at the non-overlapped regions was evaluated. This process was repeated for each candidate threshold. Then, the threshold with the largest gap between the two groups and a balanced number of individuals (at least 20 individuals per group) was selected as an optimum candidate threshold. If there was more than one selected threshold for a PSG parameter, the one with a physiological meaning was selected. These three criteria to choose a threshold reduced the number of PSG parameters to be predicted from 78 to 51 . 


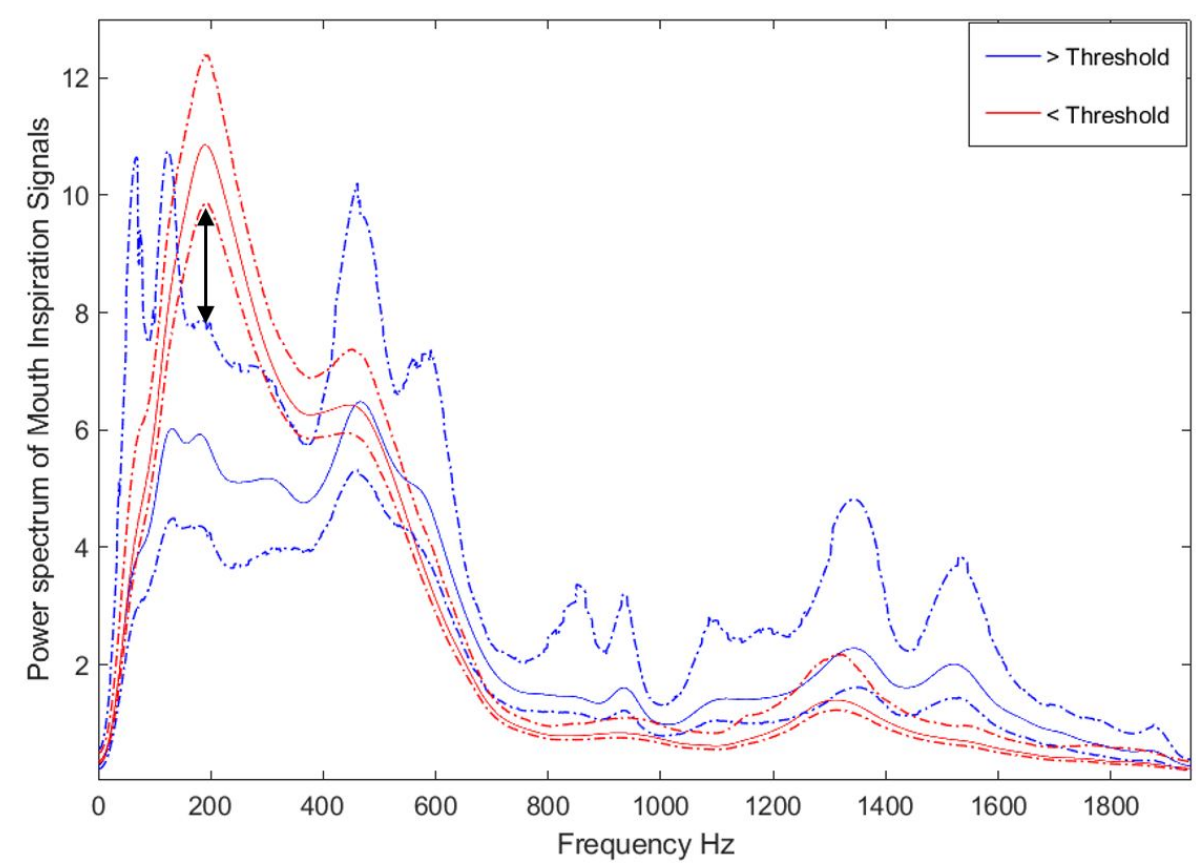

Figure 2. The average power spectrum of the signal recorded from mouth inspiration. Dotted lines represent the $95 \%$ confidence interval. Red and blue color curves represent the groups with more and less than a threshold, respectively. The arrow shows a large gap beyond the confidence interval between the two groups.

Figure 3 shows an example of the gap values for different thresholds for a PSG parameter (i.e., the mean $\mathrm{SpO} 2 \%$ during total TST); thresholds $<92 \%$ or $>95 \%$ had high average gap values, but the number of individuals per group was less than 20 . The physiological meanings for different PSG parameters were discussed in the literature, in particular, the percentage of stages III and REM of sleep [18], total arousal index [19], mean SpO2\% [20], and oxygen desaturation (de-SpO2) index [21].

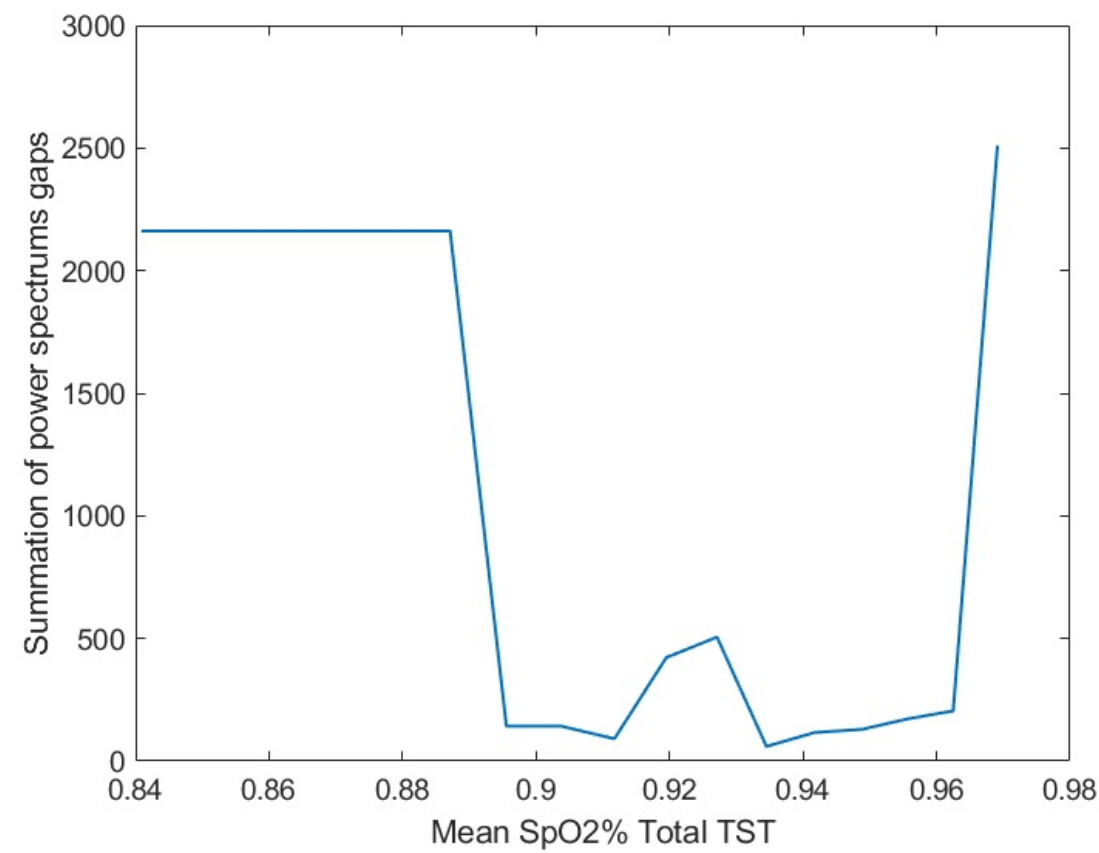

Figure 3. The average gap values among different breathing maneuvers for different thresholds of the mean $\mathrm{SpO} 2 \%$ total TST. 
Dataset segmentation-The dataset was divided into five folds of blind testing datasets; one-fold consisted of $20 \%$ of the data above the PSG parameter threshold and $20 \%$ of the data below the threshold. Taking the $20 \%$ above and below the threshold guaranteed no significant difference between the testing and training datasets, and it also made the two datasets comparable with the same severity level. Each fold of testing ( $20 \%$ of the data) with its corresponding training and validation dataset $(80 \%$ of the whole dataset) were separately analyzed. Herein, we call them the five data configurations.

Feature selection-The sound features were adopted from different anthropometric groups of features used in our previous work [8]; therefore, some features might be highly correlated with one another and need to be filtered. Thus, for a PSG parameter, the correlation coefficient (CC) between every pair of sound features was calculated; if the CC was $\geq 0.95$, we calculated the CC between each of the two features and the PSG parameter, and the feature with the lowest CC was subsequently removed. This approach guarantees removing similar features. Then, each feature's data were divided into two groups based on the selected PSG threshold. The outliers for each feature were identified in each PSG severity group and then removed [22]; this process is delineated in our previous work [8]. The significance between the two severity groups was calculated, and only features with a $p$-value $\leq 0.05$ were selected. Therefore, each PSG parameter has its own significant set of features. These processes were separately applied to the five data configurations.

\subsection{Bilinear Modeling and Correlation Analysis}

A bilinear polynomial model of three variables (a three-feature combination of sound and anthropometric features) was generated for each PSG parameter; see Equation (1) for the polynomial model (X, Y, and $\mathrm{Z}$ are three features, and a is a constant). We did not use higher-order models to avoid overfitting due to the small sample size. For simplicity, we mentioned only the methodology using the three-feature combination, but the presented results are for three-, four-, and five-feature combinations. The model was created from each three-feature combination using the multiple linear regression function in Matlab ${ }^{\mathrm{TM}} 2019$ [23]. Each model produced a new array of values representing the PSG parameter. The correlation coefficient using Spearman [24] was evaluated between the predicted (model) and actual values (extracted from the PSG report).

$$
\text { Predicted PSG parameter }=a_{1} X Y+a_{2} X Z+a_{3} Y Z+a_{4} X+a_{5} Y+a_{6} Z+a_{7}
$$

The number of models was then reduced based on their correlation with the actual PSG parameter and the percentage of overlap between different PSG parameter severity groups; the first 20 models with the highest correlation and the lowest overlap percentage were selected for the classification process; see [25] for more details on this step.

\subsection{Classification and Prediction of PSG Parameters}

Breathing sound signals are stochastic signals in nature, and the OSA disorder has many confounding factors that affect breathing sounds, which increases the heterogeneity and complexity of data. Therefore, it is required to have a classification process with multiple thresholds for each feature to overcome the complexity and heterogeneity; such a classification approach is possible in the random-forest (RF) algorithm [26]. In addition, RF does not require a normality assumption. Therefore, a classification process was performed using a two-class RF classifier with 1200 iterations using the selected threshold and the selected models. For each iteration, the classifier used $65 \%$ of the training/validation data for training and the other $35 \%$ for validation. Then, the validated classifier was used to classify the blind testing dataset. The input for the classifier was the predicted PSG parameter using the evaluated bilinear model; thus, it was a one-feature RF classifier. The models (three-, four-, five-feature combinations) that provided the validation and testing accuracies $\geq 70 \%$ and validation and testing sensitivities and specificities $\geq 50 \%$ were selected as the best models. Then, we combined the classifier outcomes of three models to find the best combinations covering most of the dataset while providing the 
highest classification performance. The final classification was based on the majority voting of each individual classifier; see [25] for more details on this section. The classification process was separately applied on the five data configurations, and then their results were averaged for each PSG parameter separately.

\section{Results}

The selected anthropometric (F1-F6) and sound features (F7-F79) for the analyses are listed in Table 1. The anthropometric characteristics of the study sample are presented in Table 2.

Table 2. Anthropometric information characteristics of the study.

\begin{tabular}{ccc}
\hline & Non-OSA (AHI $<\mathbf{1 5 )} \boldsymbol{n = 8 0}$ & OSA (AHI $\geq \mathbf{1 5}) \boldsymbol{n}=\mathbf{6 5}$ \\
\hline AHI, events $/ \mathrm{h}$, median (IQR) & $2.9(0.33-6.68)$ & $29.4(20.1-57.3)$ \\
\hline Age, years, median (IQR) & $50(41-57)$ & $51(43-59)$ \\
\hline Sex, $\mathrm{n}(\%)$ & & \\
Female & $43(53.8)$ & $17(26.2)$ \\
Male & $37(46.3)$ & $48(73.8)$ \\
\hline BMI, $\mathrm{kg} / \mathrm{m}^{2}$, median (IQR) & $31.2(26.9-35.7)$ & $34.8(30.2-39.8)$ \\
\hline NC, cm, mean (SD) & $39.87(4.98)$ & $44.37(3.74)$ \\
\hline MpS, n (\%) & & \\
I & $45(56.3)$ & $23(23.1)$ \\
II & $21(26.3)$ & $19(29.4)$ \\
III & $11(13.8)$ & $8(12.3)$ \\
\hline IV & $3(3.8)$ &
\end{tabular}

OSA is obstructive sleep apnea, NC is neck circumference, MpS is Mallampati score, IQR is interquartile range, and SD is standard deviation. AHI is Apnea-hypopnea index; BMI is body mass index.

Table 3 shows the most significant and useful correlation coefficients (a maximum of 0.56) between the PSG parameters and anthropometric/sound features. Only those PSG parameters that resulted in high testing results are presented. Males had a higher number of apneic events than females. The increase in neck circumference and/or BMI was associated with an increase in the number of obstructive events and arousals. A high arousal index and apnea/hypopnea index were associated with an increase in the power of the high-frequency components of tracheal breathing sounds. Furthermore, a high oxygen desaturation index was associated with a high first resonance frequency in breathing sounds. Figure 4 shows the scatter plots of four PSG parameters with their correlated features.

Table 4 shows the bilinear model feature combinations with the highest overall correlation coefficients with the PSG parameters. Only the highest two model coefficients are represented in parentheses right to the relevant feature, and the bolded feature is the one with the highest contribution to the constructed model. Furthermore, F1 scores and testing classification accuracies of the blind testing dataset using the corresponding models to predict the PSG parameters are presented. The constructed models had correlation coefficients $>0.8$ with nine OSA severity parameters. The supine arousal index had a correlation coefficient of 0.8 with the F5-F6-F37-F46-F74 bilinear model, and the average power of high frequencies for the nose inspiratory signal was the main parameter in the bilinear model. The hypopnea index had a correlation coefficient of 0.82 with the F4-F5F12-F26-F38 bilinear model, and neck circumference was the main parameter in the bilinear model. The testing classification accuracy and F1 score were $92 \%$ and $95 \%$, respectively, for predicting AHI measured in the supine position with a threshold of 15 . The scatter plots of the four previously mentioned PSG parameters with their highly correlated bilinear equation models are shown in Figure 5. These plots show the enhancement in correlation coefficients and linear relationships compared to those shown in Figure 4. 
Table 3. The spearman correlation coefficients between the anthropometric/sound feature and the PSG parameters.

\begin{tabular}{|c|c|c|c|}
\hline PSG\# & PSG Parameter & Anthropometric and Sound Feature & Correlation Coefficient \\
\hline PSG1 & Stage 3 Duration $\%$ & $\mathrm{~F} 2$ & -0.35 \\
\hline PSG2 & EEG Total Arousal Index & F11/F37/F8 & $0.35 / 0.33 /-0.31$ \\
\hline PSG3 & Total Arousal Index & F5/F11/F37 & $0.41 / 0.35 / 0.33$ \\
\hline PSG4 & Supine Arousal Index & F5/F37/F46/F53 & $0.46 / 0.36 / 0.31 / 0.31$ \\
\hline PSG5 & Non-Supine Arousal Index & $\mathrm{F} 1 / \mathrm{F} 4 / \mathrm{F} 10 / \mathrm{F} 69$ & $0.37 / 0.36 / 0.36 / 0.36$ \\
\hline PSG6 & Supine Sleep \% & $\mathrm{F} 1 / \mathrm{F} 17$ & $-0.29 / 0.29$ \\
\hline PSG7 & Apnea Total Index & F3/F5/F51 & $-0.5 / 0.41 /-0.38$ \\
\hline PSG8 & Apnea Non-REM Index & F3/F5/F49 & $-0.51 / 0.46 /-0.39$ \\
\hline PSG9 & Apnea Total Index (No Central) & F3/F5/F51 & $-0.44 / 0.38 /-0.33$ \\
\hline PSG10 & Apnea Non-REM Index (No Central) & F3/F5/F49/F37 & $-0.45 / 0.43 /-0.37 / 0.36$ \\
\hline PSG11 & Hypopnea Total Index & $\mathrm{F} 5 / \mathrm{F} 26 / \mathrm{F} 4 / \mathrm{F} 37 / \mathrm{F} 49$ & $0.54 /-0.45 / 0.41 / 0.4 /-0.4$ \\
\hline PSG12 & Hypopnea REM Index & F31 & -0.34 \\
\hline PSG13 & Hypopnea Non-REM Index & F5/F4/F37/F57 & $0.53 / 0.41 / 0.41 / 0.4$ \\
\hline PSG14 & Hypopnea Total Index (No Central) & F5/F32/F4/F49 & $0.54 /-0.43 / 0.41 /-0.4$ \\
\hline PSG15 & Hypopnea REM Index (No Central) & $\mathrm{F} 1 / \mathrm{F} 20 / \mathrm{F} 4 / \mathrm{F} 6$ & $0.3 / 0.28 / 0.27 / 0.26$ \\
\hline PSG16 & Hypopnea Non-REM Index (No Central) & F5/F4/F37/F49 & $0.53 / 0.41 / 0.41 /-0.4$ \\
\hline PSG17 & Supine Sleep Obs. Apnea & $\mathrm{F} 5 / \mathrm{F} 3 / 49$ & $0.43 /-0.43 /-0.36$ \\
\hline PSG18 & Supine Sleep Obs. Hypopnea & F5/F26/F49 & $0.51 /-0.38 /-0.37$ \\
\hline PSG19 & Supine Sleep A + H (with central) & F5/F37/F57/F10 & $0.6 / 0.41 / 0.38 / 0.36$ \\
\hline PSG20 & Supine Sleep A + H (without central) & F5/F57/F26/F49 & $0.55 / 0.42 /-0.4 /-0.41$ \\
\hline PSG21 & Left-side Sleep Obs. Hypopnea & $\mathrm{F} 5 / \mathrm{F} 1 / 61 / \mathrm{F} 32$ & $0.45 / 0.42 /-0.38 /-0.38$ \\
\hline PSG22 & Left-side Sleep A + H (with central) & $\mathrm{F} 5 / \mathrm{F} 1 / \mathrm{F} 32 / \mathrm{F} 4$ & $0.45 / 0.4 /-0.39 / 0.36$ \\
\hline PSG23 & Left-side Sleep A + H (without central) & $\mathrm{F} 1 / \mathrm{F} 32 / \mathrm{F} 4$ & $0.4 /-0.4 / 0.37$ \\
\hline PSG24 & Right-side Sleep Obs. Hypopnea & F26/F69/F1/F38 & $-0.44 /-0.43 / 0.41 / 0.41$ \\
\hline PSG25 & Right-side Sleep A + H (with central) & F8/F5/F67/F27 & $-0.43 / 0.43 / 0.41 / 0.4$ \\
\hline PSG26 & Right-side Sleep A + H (without central) & F21/F26/F5/F8/F9 & $-0.43 /-0.44 / 0.42 /-0.43 /-0.42$ \\
\hline PSG27 & Total AHI (With Central) & F5/F21/F25/F54 & $0.56 /-0.41 / 0.4 / 0.4$ \\
\hline PSG28 & Total AHI (Without Central) & F5/F37/F57/F49 & $0.54 / 0.43 / 0.43 /-0.42$ \\
\hline PSG29 & De-SpO2 Index Total & $\mathrm{F} 5 / \mathrm{F} 60 / \mathrm{F} 37 / \mathrm{F} 1$ & $0.56 /-0.44 / 0.42 / 0.4$ \\
\hline PSG30 & De-SpO2 Index Non-REM & $\mathrm{F} 5 / \mathrm{F} 4 / \mathrm{F} 21 / \mathrm{F} 37$ & $0.55 / 0.43 /-0.41 / 0.42$ \\
\hline PSG31 & De-SpO2 Index REM & F1/F5/F8/F23 & $0.41 / 0.39 /-0.38 / 0.38$ \\
\hline PSG32 & Mean SpO2\% Total TST & $\mathrm{F} 1 / \mathrm{F} 47 / \mathrm{F} 4$ & $-0.4 /-0.39 /-0.36$ \\
\hline PSG33 & Mean SpO2\% Supine & F47 & -0.32 \\
\hline PSG34 & Mean SpO2\% Non-Supine & $\mathrm{F} 1 / \mathrm{F} 4 / \mathrm{F} 47 / \mathrm{F} 37$ & $-0.47 /-0.43 /-0.4 /-0.39$ \\
\hline PSG35 & REM Latency/TST & F28/F29 & $-0.29 / 0.25$ \\
\hline PSG36 & Wake after sleep Onset/TST & F2 & 0.39 \\
\hline
\end{tabular}

F: feature label, BMI: body mass index, NC: neck circumference, A+H: apnea and hypopnea, and REM: rapid eye movement. PSG\#: PSG parameter number. SpO2: Oxygen saturation. De-SpO2: Oxygen desaturation. EEG: Electroencephalogram. TST: total time of sleep. 

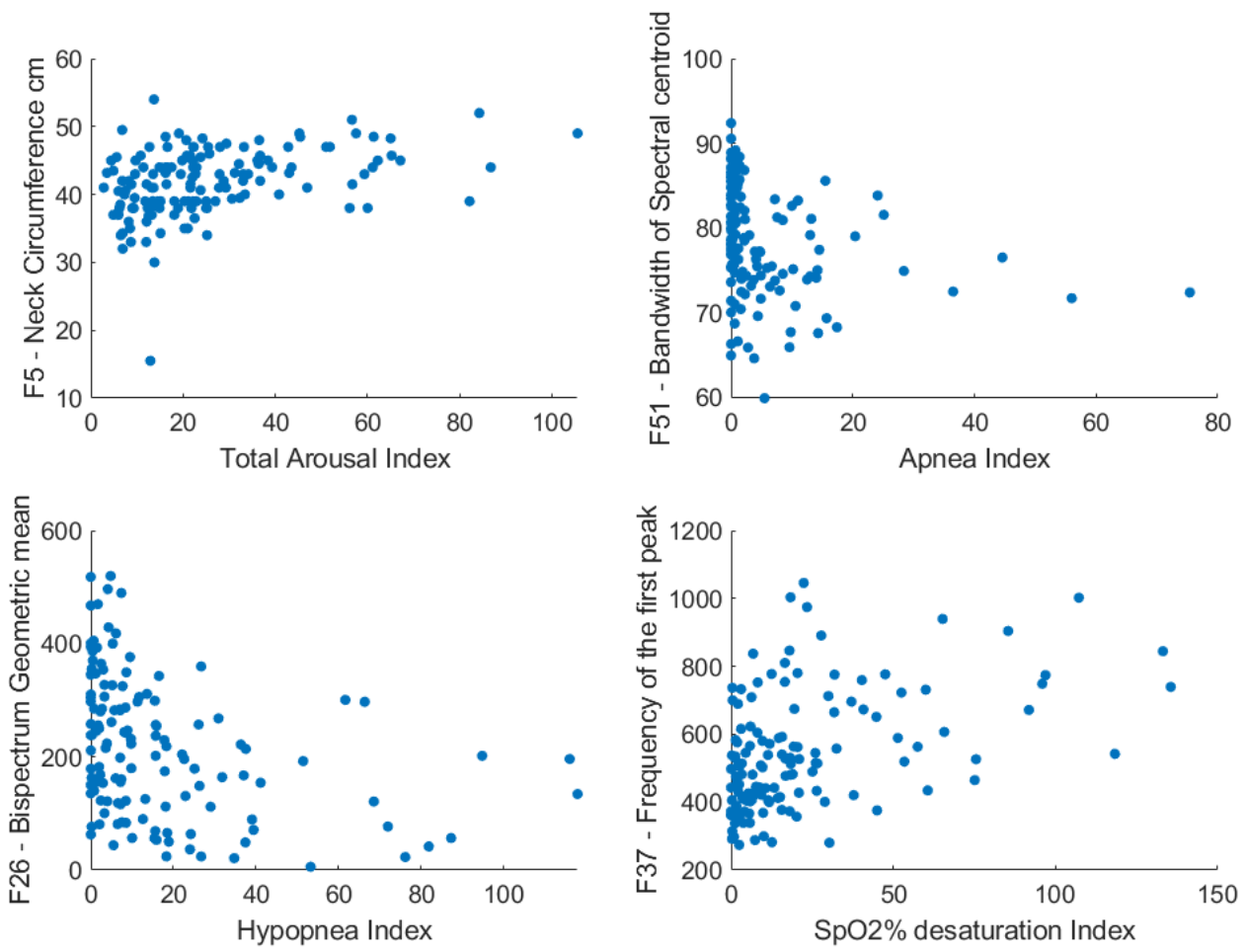

Figure 4. The scatter plots between four PSG parameters and the anthropometric and sound features with the highest correlation coefficients with them. F: feature label.
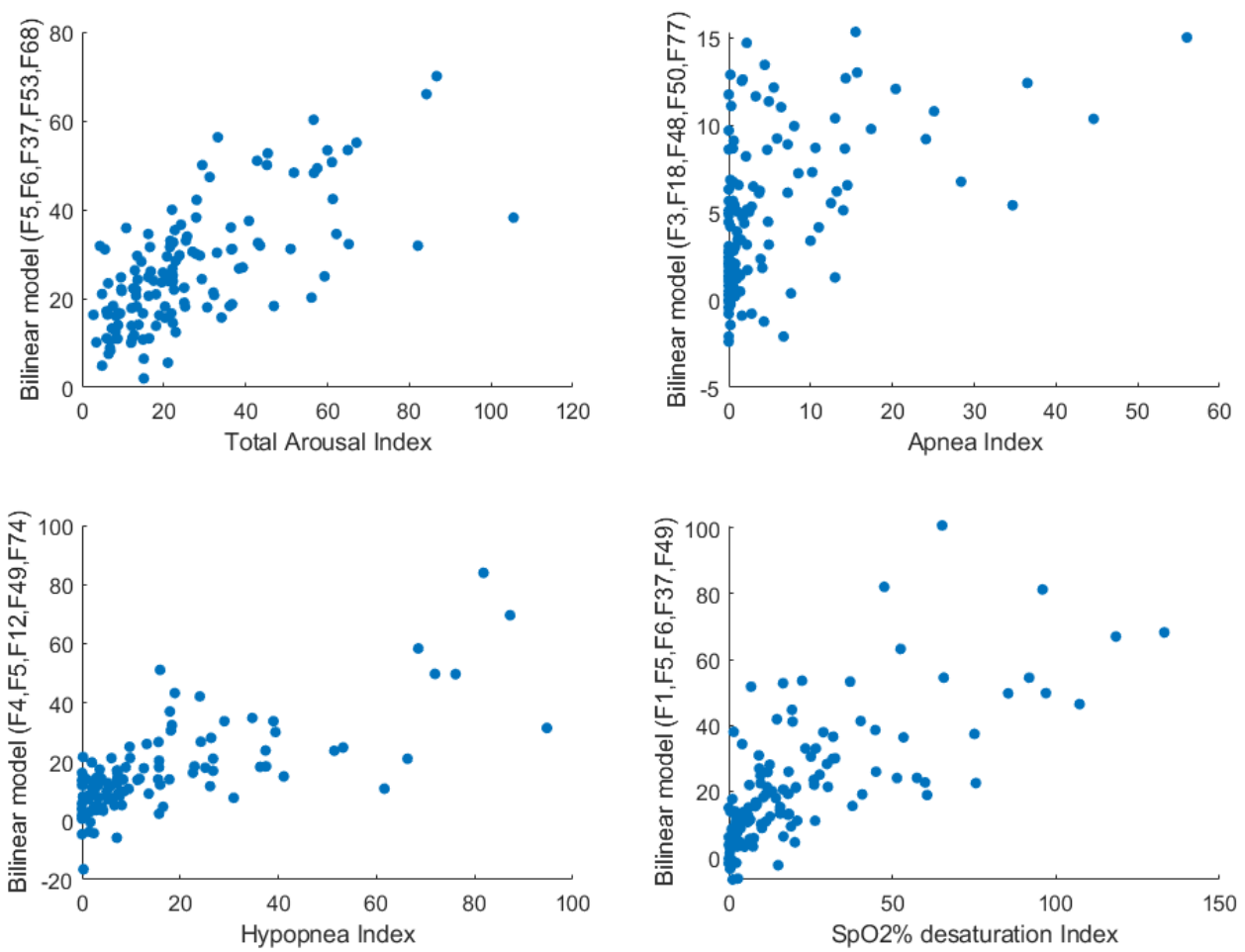

Figure 5. The scatter plots between four PSG parameters and the outcomes of the bilinear model. F: feature label. 
Table 4. The correlation coefficients between the PSG parameters and the outcomes of the bilinear model for the entire dataset, as well as their blind testing F1 scores and classification accuracies.

\begin{tabular}{|c|c|c|c|c|}
\hline PSG\# & Feature Combination & $\mathrm{CC}$ & F1-Score & $\mathrm{ACC} \%$ \\
\hline PSG1 & F2 F3(0.31) F46(-0.14) F74 F77 & 0.63 & 0.63 & 63 \\
\hline PSG2 & F8(7.3) F12 F46(110) F49 F56 & 0.7 & 0.82 & 80 \\
\hline PSG3 & F5(-31) F6(246) F37 F53 F68 & 0.74 & 0.76 & 81 \\
\hline PSG4 & F5 F6(-21) F37 F46(86) F74 & 0.8 & 0.62 & 64 \\
\hline PSG5 & F1 F10 F49(0.73) F56(-3.2) F72 & 0.71 & 0.62 & 74 \\
\hline PSG6 & F2 F4 F33(0.07) F35 F46(2.3) & 0.64 & 0.64 & 64 \\
\hline PSG7 & F3(106) F18 F48(-1367) F50 F77 & 0.75 & 0.74 & 71 \\
\hline PSG8 & F3(8.4) F37 F48(-562) F49 F51 & 0.72 & 0.67 & 71 \\
\hline PSG9 & F3 F19 F46(30.7) F48(-342) F77 & 0.69 & 0.6 & 67 \\
\hline PSG10 & F3(30.7) F6(64.4) F19 F53 F78 & 0.72 & 0.63 & 68 \\
\hline PSG11 & F4(2.6) F5(13.4) F12 F26 F38 & 0.82 & 0.83 & 82 \\
\hline PSG12 & F4(-5.5) F5 F12(-14) F49 F74 & 0.72 & 0.67 & 79 \\
\hline PSG13 & F4 F5(17.1) F11 F49(-5.5) F52 & 0.81 & 0.6 & 62 \\
\hline PSG14 & F1(-7.2) F4(5.5) F5 F12 F15 & 0.76 & 0.78 & 78 \\
\hline PSG15 & F1(-0.96) F29 F30 F49(-1.3) & 0.59 & 0.6 & 78 \\
\hline PSG16 & F5 F6(36.3) F10 F46(288) F53 & 0.78 & 0.8 & 79 \\
\hline PSG17 & F3 F46(11.1) F57(79.1) F66 F77 & 0.75 & 0.73 & 77 \\
\hline PSG18 & F4 F5(9.9) F10 F17 F49(2.1) & 0.79 & 0.62 & 71 \\
\hline PSG19 & F5(3.1) F38 F57(-730) F68 F71 & 0.79 & 0.95 & 92 \\
\hline PSG20 & F5(32.3) F6(-99) F11 F50 F74 & 0.83 & 0.86 & 85 \\
\hline PSG21 & F4(3.9) F5(-10.8) F24 F42 & 0.64 & 0.78 & 74 \\
\hline PSG22 & F4 F5(-3.3) F6(33.48) F32 F66 & 0.73 & 0.67 & 69 \\
\hline PSG23 & F1 F6(473) F32(-80.5) F49 F53 & 0.73 & 0.63 & 67 \\
\hline PSG24 & F6(-15.3) F8(3.9) F10 F38 F41 & 0.81 & 0.77 & 73 \\
\hline PSG25 & F5(-76) F6(113) F19 F54 F77 & 0.79 & 0.67 & 60 \\
\hline PSG26 & F6 F9(-18) F26 F38 F46(-23.5) & 0.84 & 0.73 & 72 \\
\hline PSG27 & F5(-161) 21 F25(-2550) F38 F51 & 0.81 & 0.67 & 75 \\
\hline PSG28 & F5 F11 F25(-1889) F57(-4046) F71 & 0.8 & 0.63 & 67 \\
\hline PSG29 & F1 F5(4.4) F6(-8.4) F37 F49 & 0.77 & 0.63 & 73 \\
\hline PSG30 & F5(12.73) F21 F53 F65(-89.5) F77 & 0.78 & 0.84 & 80 \\
\hline PSG31 & F6(44) F16 F29 F30 F65(24.7) & 0.8 & 0.89 & 92 \\
\hline PSG32 & F1(-0.005) F24(0.0009) F29 F51 & 0.65 & 0.62 & 58 \\
\hline PSG33 & F18 F28 F29 F47(0.15) F68(-0.0004) & 0.63 & 0.72 & 68 \\
\hline PSG34 & F1(0.0157) F2(-0.006) F31 F37 F53 & 0.71 & 0.8 & 72 \\
\hline PSG35 & F1(0.22) F2 F28 F32(-0.13) F36 & 0.63 & 0.74 & 74 \\
\hline PSG36 & F2(-0.75) F22 F39 F46(1.78) F76 & 0.55 & 0.7 & 65 \\
\hline
\end{tabular}

F: feature label, PSG\#: PSG parameter number, CC: correlation coefficient with the outcomes of the bilinear model, and ACC: testing classification accuracy. Feature coefficient in the model is represented in the parentheses, and bolded features are the ones with the highest contribution to the constructed model.

Figure 6 shows the average inspiratory/expiratory power spectra during mouth/nose breathing of four PSG parameter groups. The corresponding sound spectra to each PSG parameter are represented using two curves (i.e., blue and red) with their $95 \%$ confidence 
intervals (dotted lines). The blue curve represents the average curves of individuals with the PSG parameters less than the selected threshold, while the red curve represents the average curves of individuals with the PSG parameters more than the selected threshold. There is a big gap in the range of $100-300 \mathrm{~Hz}$ between the mouth inspiratory power spectra of patients with a supine arousal index of more than 30 and less than 30.
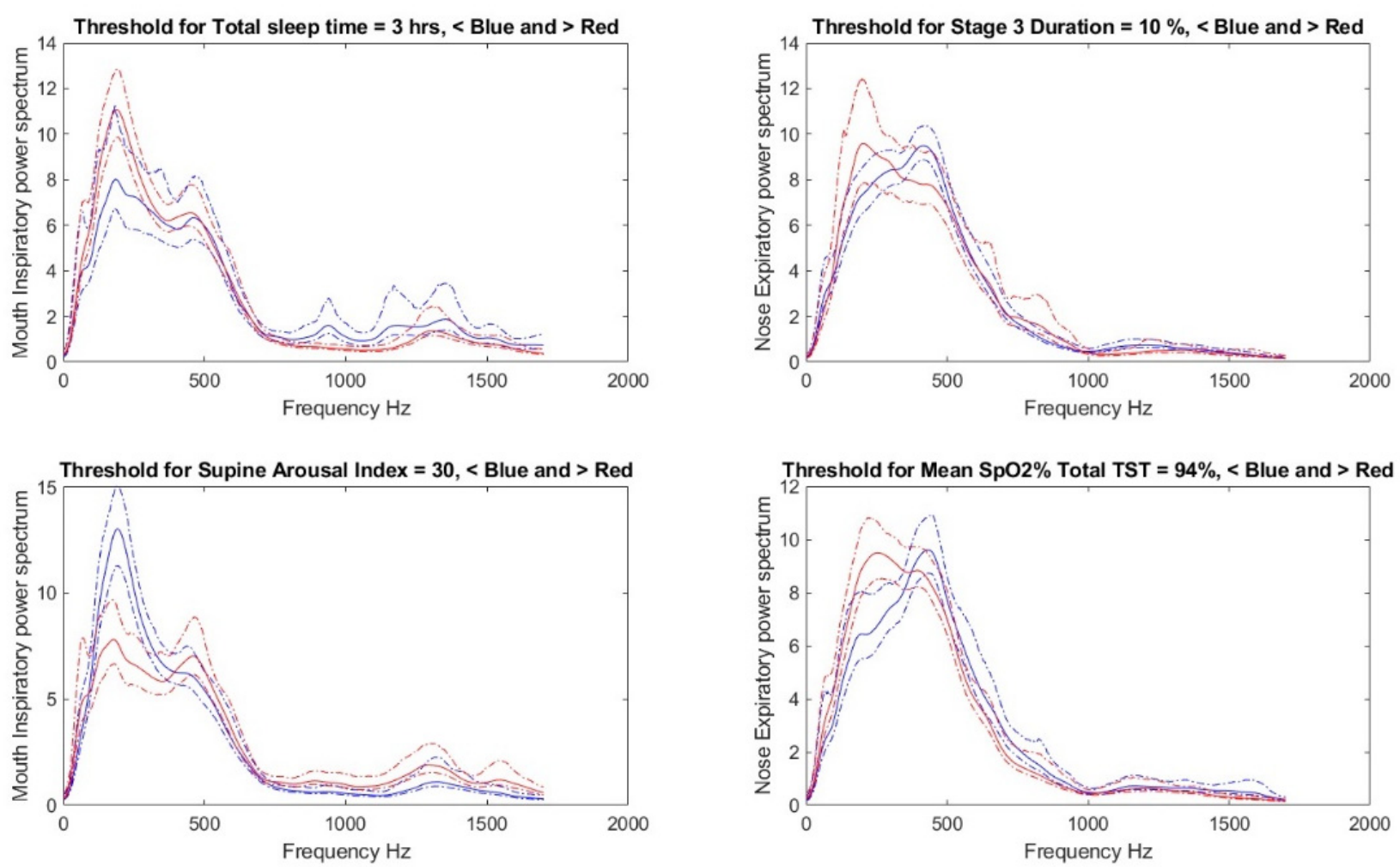

Figure 6. The average power spectrum of the signal recorded from mouth inspiration. Dotted lines represent the $95 \%$ confidence interval. Red and blue color curves represent the groups with more and less than the threshold, respectively.

On average, a healthy adults' sleep duration is 5\% in stage $1,50 \%$ in stage $2,20 \%$ in stage 3, and 20-25\% in REM [18]. We used thresholds of $10 \%$ for stage 3 sleep as an indicator of abnormal sleep duration. The total arousal index threshold depends on age; it is 10 for young, 15-20 for middle-aged, and 25 for elders [19]. We used 25 as the threshold for predicting the total arousal index, as it had more separability in the power spectra of the training dataset ( $80 \%$ of the data). Furthermore, it has been mentioned that a normal mean $\mathrm{SpO} 2 \%$ is between 93\% and98\% [20]; thus, we used 93-94\% as a threshold of the beginning of an abnormal percentage. Moreover, de-SPO2 index is a value usually between 0 to 135, and it is highly correlated with AHI [21]; thus, we used 15 as the threshold for de-SPO2 index similar to an AHI of 15.

Table 5 shows the selected thresholds for the PSG parameters. The presented PSG parameters are the ones with average classification accuracies of more than $70 \%$ and average classification sensitivities and specificities of more than $60 \%$. Table 5 also shows the average validation and blind testing classification accuracies, sensitivities, specificities, and F1 scores using the outcomes of the bilinear equations for the five dataset configurations. The proposed method resulted in a blind testing classification accuracy of up to $88.8 \%$. Predicting the obstructive hypopnea index measured on the supine position with a threshold of 15 resulted in $81.6 \%$ blind testing accuracy with $86.1 \%$ validation accuracy and comparable and high F1 scores for both the validation and blind testing processes. In addition, many PSG parameters were predicted with reasonable and comparable accuracies, sensitivities, and specificities. 
Table 5. The average validation and testing accuracies for classifying data around the selected threshold using the outcomes of the bilinear models.

\begin{tabular}{|c|c|c|c|c|c|c|c|c|c|}
\hline PSG\# & Threshold & VF1 & VCA $\%$ & VSpec\% & VSens \% & TF1 & ТCA \% & TSpec\% & TSens $\%$ \\
\hline PSG1 & $10 \%$ & 0.66 & 80.7 & 88.9 & 61.9 & 0.73 & 78.2 & 80.1 & 73.8 \\
\hline PSG2 & 15 & 0.80 & 75.3 & 69.1 & 79.2 & 0.83 & 81.6 & 78.0 & 83.8 \\
\hline PSG3 & 25 & 0.65 & 74.6 & 83.9 & 60.6 & 0.66 & 73.6 & 81.6 & 61.7 \\
\hline PSG4 & 30 & 0.75 & 76.8 & 78.7 & 74.5 & 0.72 & 73.4 & 73.7 & 73.7 \\
\hline PSG5 & 20 & 0.70 & 76.2 & 81.8 & 67.9 & 0.76 & 84.7 & 97.8 & 63.3 \\
\hline PSG6 & $30 \%$ & 0.79 & 74.9 & 63.1 & 83.7 & 0.75 & 72.4 & 76.4 & 70.5 \\
\hline PSG7 & 1 & 0.81 & 80.8 & 80.6 & 81.1 & 0.83 & 82.9 & 82.1 & 84.0 \\
\hline PSG8 & 1 & 0.79 & 82.3 & 88.7 & 74.1 & 0.73 & 80.7 & 85.4 & 70.1 \\
\hline PSG9 & 3.5 & 0.70 & 83.4 & 90.4 & 66.6 & 0.76 & 85.6 & 89.7 & 78.1 \\
\hline PSG10 & 0.5 & 0.76 & 77.9 & 80.7 & 74.8 & 0.79 & 80.6 & 79.6 & 82.6 \\
\hline PSG11 & 10 & 0.78 & 83.0 & 88.5 & 75.0 & 0.74 & 80.4 & 82.2 & 76.4 \\
\hline PSG12 & 20 & 0.70 & 77.8 & 84.6 & 67.0 & 0.68 & 79.5 & 91.3 & 60.6 \\
\hline PSG13 & 7.5 & 0.83 & 84.5 & 86.7 & 81.9 & 0.78 & 80.4 & 80.1 & 80.2 \\
\hline PSG14 & 5 & 0.87 & 82.8 & 66.9 & 91.6 & 0.90 & 88.8 & 82.5 & 94.7 \\
\hline PSG15 & 20 & 0.67 & 76.2 & 82.0 & 66.3 & 0.68 & 80.6 & 89.6 & 62.5 \\
\hline PSG16 & 5 & 0.83 & 80.7 & 77.2 & 83.5 & 0.83 & 82.6 & 77.7 & 90.3 \\
\hline PSG17 & 1 & 0.77 & 77.1 & 80.1 & 74.1 & 0.77 & 79.9 & 85.6 & 74.5 \\
\hline PSG18 & 15 & 0.86 & 86.1 & 85.3 & 86.9 & 0.78 & 81.6 & 82.1 & 79.8 \\
\hline PSG19 & 15 & 0.87 & 85.7 & 83.9 & 86.9 & 0.82 & 79.7 & 71.8 & 86.3 \\
\hline PSG20 & 20 & 0.84 & 83.4 & 80.6 & 85.8 & 0.81 & 81.4 & 79.4 & 83.4 \\
\hline PSG21 & 2.5 & 0.76 & 78.8 & 82.1 & 74.1 & 0.78 & 80.4 & 78.5 & 84.8 \\
\hline PSG22 & 7 & 0.76 & 84.4 & 92.1 & 70.3 & 0.71 & 80.2 & 85.9 & 69.8 \\
\hline PSG23 & 4 & 0.74 & 80.1 & 87.1 & 69.6 & 0.75 & 79.5 & 78.0 & 79.2 \\
\hline PSG24 & 1 & 0.84 & 81.6 & 80.3 & 82.6 & 0.82 & 80.4 & 77.5 & 82.5 \\
\hline PSG25 & 0.5 & 0.87 & 82.5 & 71.9 & 88.2 & 0.91 & 88.4 & 94.3 & 86.9 \\
\hline PSG26 & 0.5 & 0.86 & 82.2 & 75.6 & 86.1 & 0.87 & 83.7 & 85.0 & 84.2 \\
\hline PSG27 & 15 & 0.77 & 78.7 & 81.2 & 75.8 & 0.78 & 82.4 & 85.0 & 79.3 \\
\hline PSG28 & 15 & 0.76 & 80.5 & 87.1 & 71.7 & 0.75 & 80.5 & 83.9 & 74.5 \\
\hline PSG29 & 15 & 0.75 & 77.2 & 81.1 & 72.8 & 0.78 & 82.0 & 84.5 & 78.7 \\
\hline PSG30 & 5 & 0.87 & 83.0 & 70.7 & 89.5 & 0.89 & 86.5 & 77.9 & 91.6 \\
\hline PSG31 & 25 & 0.78 & 83.3 & 91.5 & 71.5 & 0.77 & 82.5 & 83.9 & 80.0 \\
\hline PSG32 & $94 \%$ & 0.72 & 70.5 & 68.7 & 72.0 & 0.74 & 71.5 & 68.6 & 75.4 \\
\hline PSG33 & $94 \%$ & 0.69 & 72.3 & 77.0 & 66.6 & 0.78 & 77.8 & 74.7 & 81.9 \\
\hline PSG34 & $93 \%$ & 0.88 & 81.8 & 60.1 & 90.9 & 0.92 & 87.8 & 77.1 & 92.3 \\
\hline PSG35 & 0.45 & 0.78 & 72.4 & 64.3 & 77.2 & 0.88 & 86.5 & 100 & 80.6 \\
\hline PSG36 & 0.15 & 0.78 & 72.4 & 60.0 & 79.8 & 0.79 & 74.1 & 63.9 & 80.8 \\
\hline
\end{tabular}

F: feature label, PSG\#: PSG parameter number, V: validation, T: blind testing, CA: classification accuracy using the outcomes of the bilinear models, F1: F1 score, Spec: specificity, and Sens: sensitivity.

\section{Discussion}

The results of this study show the possibility to predict AHI and other OSA severity parameters with high precision during wakefulness by using only anthropometric informa- 
tion and five cycles of breathing sounds. Thus, the proposed methodology shows great potentials for a quick, informative, and highly reliable OSA screening tool.

Tracheal breathing sounds' features showed significant correlations with some of the PSG parameters (Table 3). Feature 37 showed a high positive correlation with the total arousal index. This suggests that individuals with high arousal index have a high first frequency peak. The peaks at higher frequency represent stiffness, and that implies high UA dilator muscle activities [15]. Feature 47 was found to be high in individuals with a low average $\mathrm{SpO} \%$ during sleep. This indicates high power at low frequencies among those with high $\mathrm{SpO} \%$; this is also congruent with our previous findings [15] as individuals with $\mathrm{AHI}<15$ have high power in the low-frequency components. Furthermore, this high power indicates a wide UA and, thus, low UA resistance.

As shown in Table 1, most of the selected sound features were extracted from inspiratory breathing; inspiration is an active process, hence, the UA muscles are active. This finding was expected because the UA muscle atrophy is one of the causes of the UA collapse leading to OSA. Thus, we expected the characteristic features of the breathing sounds in relation to OSA to be mostly selected during the inspiratory phase. This is also congruent with our previous studies [15,27], in which the characteristic breathing sounds in correlation with AHI were mostly selected from an inspiratory phase. Furthermore, PSG parameters were correlated with various sound features, which were extracted from linear and non-linear algorithms and from time and frequency domains. This indicates the complexity of the UA structure and the ability of breathing sound features to identify different UA characteristics.

Using models of bilinear equations resulted in better correlations with the PSG parameters than using only one-feature combinations (see Figure 5 and Table 4). Based on the selected thresholds and using the selected model combinations, we were able to reach up to $86.1 \%$ and $88.8 \%$ average validation and blind testing classification accuracy (VCA and TCA) with comparable sensitivities and specificities. Using five different data configurations and executing the entire process for each configuration ensured the unbiased reliability of the extracted models. The F1 scores, sensitivities, and specificities show the ability of the classifiers to have unbiased decisions. Therefore, the results demonstrate the ability to predict the PSG parameters with high confidence using combinations of anthropometric and breathing sounds features. The proposed method could be used as a fast-diagnosing tool that predicts several OSA severity parameters, and could benefit sleep physicians, anesthesiologists, and dentists.

Anthropometric parameters such as BMI, NC, age, etc. have shown their ability to screen for OSA; however, they provide low screening specificity. Nevertheless, as shown in this study, they had significant correlations with the attributes describing the OSA disorder evaluated by a PSG assessment. Sleep efficiency (TST/total time on bed) and stage 3 duration (representing deep sleeping) have been shown to decrease with age [18]. Additionally, average $\mathrm{SpO} \%$ and REM sleep duration were shown to decrease with obesity (increasing BMI) [28]. Our data (Table 3) are congruent with the above observations. In addition, our results showed that many event indices per hour during sleep, such as total arousal index, hypopnea index, total de-SaO2 index, etc., are positively correlated with NC. Furthermore, NC was selected as a significant feature in many models predicting several PSG parameters, which indicates its importance on the OSA screening process. Therefore, the outcomes confirm the relationships between OSA risk factors and the pathophysiology of the disorder.

In our previous works, as well as the work of other researchers [8,15,29-31], tracheal breathing sound features showed a high correlation with OSA severity in terms of AHI values. Investigating the power spectra for PSG threshold determination revealed some interesting observations. For example, the selected threshold for the apnea index in this study was 1 , while it was 10 for the hypopnea index. This implies that an apneic event affects the UA much more significantly than a hypopnic event. Additionally, the selected supine arousal index threshold was 25, while it was 30 for the non-supine arousal index. 
The thresholds for hypopnea index and de-SPO2 index during non-REM sleep were lower than the threshold selected during REM sleep. Furthermore, the thresholds for obstruction indexes, in general, were higher when the patients were sleeping on their left side than those recorded when they were on their right side. These observations show a significant change in the UA structure in supine or right-side body positions and/or during non-REM sleep, and imply that the upper airway is at a higher risk if an obstruction event happens.

\section{Limitations and Future Work}

The main limitation of this study was its sample size $(n=145)$, which, given the heterogeneity of the OSA population, is not enough to represent the whole OSA population. Future studies with a larger sample size are needed to replicate and validate the claims of this paper. The thresholds were mainly selected based on the separability between the two PSG parameter groups, while having a reasonable number of individuals in each group. Therefore, it is recommended to replace some of the selected thresholds with the ones that imply high health risks. The dataset could not be matched in terms of all of the mentioned anthropometric parameters due to the small sample size. Once we have a larger sample, we will investigate predicting each PSG parameter in different anthropometric groups, the same as the AWakeOSA analysis. Another limitation is that in this study, all the features were adopted from our previous study [8], and not extracted from visualizing the spectra of each PSG parameter. In future work, we will investigate the spectra for each PSG parameter separately to extract the most prominent features and assess whether those features would be much different from the current ones. Furthermore, the PSG reports did not have any information regarding average apnea or hypopnea events' duration and average oxygen desaturation percentage duration during obstructive events. Once we resume our sleep studies in our sleep lab, we will consider recording them in our future studies, and investigate the possibility of their prediction.

\section{Conclusions}

Some PSG parameters, such as AHI, sleep duration, number of arousals, etc., are crucial to understand the pathophysiology of the OSA disorder and choose an appropriate treatment. Evaluating the parameters using simple means during wakefulness in a short time is an essential step towards an expeditious treatment; moreover, it will significantly help anesthesiologists who need to know the OSA status of their patients prior to surgery. This study is the first to investigate the prediction of the PSG parameters other than AHI during wakefulness using individuals' anthropometric information and a few minutes of breathing sound recording. The promising results of this study for predicting the PSG parameters with meaningful features may pave the way for reducing the waiting lines and providing sleep clinics with a quick and reliable OSA screening tool.

Author Contributions: A.E. conceived the experiment(s) and main data analysis, and Z.M. supervised the entire project and contributed to the writing of the paper and discussion of the results. Both authors contributed equally to writing the paper. All authors have read and agreed to the published version of the manuscript.

Funding: This research was funded by Natural Sciences and Engineering Research Council of Canada and Canadian Institutes of Health Research.

Institutional Review Board Statement: The study was conducted according to the guidelines of the Declaration of Helsinki, and approved by the Biomedical Research Ethics Board of the University of Manitoba (B2006:185, 27 October 2011).

Informed Consent Statement: An informed consent form approved by the Biomedical Research Ethics Board of the University of Manitoba was obtained from all participants of the study.

Data Availability Statement: Data are available for interested researchers from this page: https: //home.cc.umanitoba.ca/ mousavi/ftp/Data/.

Conflicts of Interest: The authors declare no conflict of interest. 


\section{Appendix A}

Table A1. Shows the extracted PSG parameters from the PSG report.

\begin{tabular}{|c|}
\hline \multirow{2}{*}{$\begin{array}{c}\text { PSG Parameter } \\
\text { Total sleep time TST }\end{array}$} \\
\hline \\
\hline Sleep Efficiency \\
\hline Total Awakenings \\
\hline Total Awakenings Index \\
\hline Stage 1 Duration \% \\
\hline Stage 2 Duration $\%$ \\
\hline Stage 3 Duration $\%$ \\
\hline Stage 4 Duration \% \\
\hline REM Duration $\%$ \\
\hline Respiratory-Related Total Arousal Index \\
\hline PLM-Related Total Arousal Index \\
\hline EEG Total Arousal Index \\
\hline Total Arousal Index \\
\hline NREM Total Arousal Index \\
\hline REM Arousal Index \\
\hline Supine Arousal Index \\
\hline Non-Supine Arousal Index \\
\hline Total Arousal + Awakenings Index \\
\hline Supine Sleep \% \\
\hline Prone Sleep \% \\
\hline Left-Side Sleep \% \\
\hline Right-Side Sleep \\
\hline Apnea Total Index \\
\hline Apnea REM Index \\
\hline Apnea Non-REM Index \\
\hline Apnea Total Index (No Central) \\
\hline Apnea REM Index (No Central) \\
\hline Apnea Non-REM Index (No Central) \\
\hline Hypopnea Total Index \\
\hline Hypopnea REM Index \\
\hline Hypopnea Non-REM Index \\
\hline Hypopnea Total Index (No Central) \\
\hline Hypopnea REM Index (No Central) \\
\hline Hypopnea Non-REM Index (No Central) \\
\hline Supine Sleep Obs. Apnea \\
\hline Supine Sleep Mix. Apnea \\
\hline Supine Sleep Cen. Apnea \\
\hline Supine Sleep Obs. Hypopnea \\
\hline Supine Sleep A+H (with central) \\
\hline
\end{tabular}


Table A1. Cont.

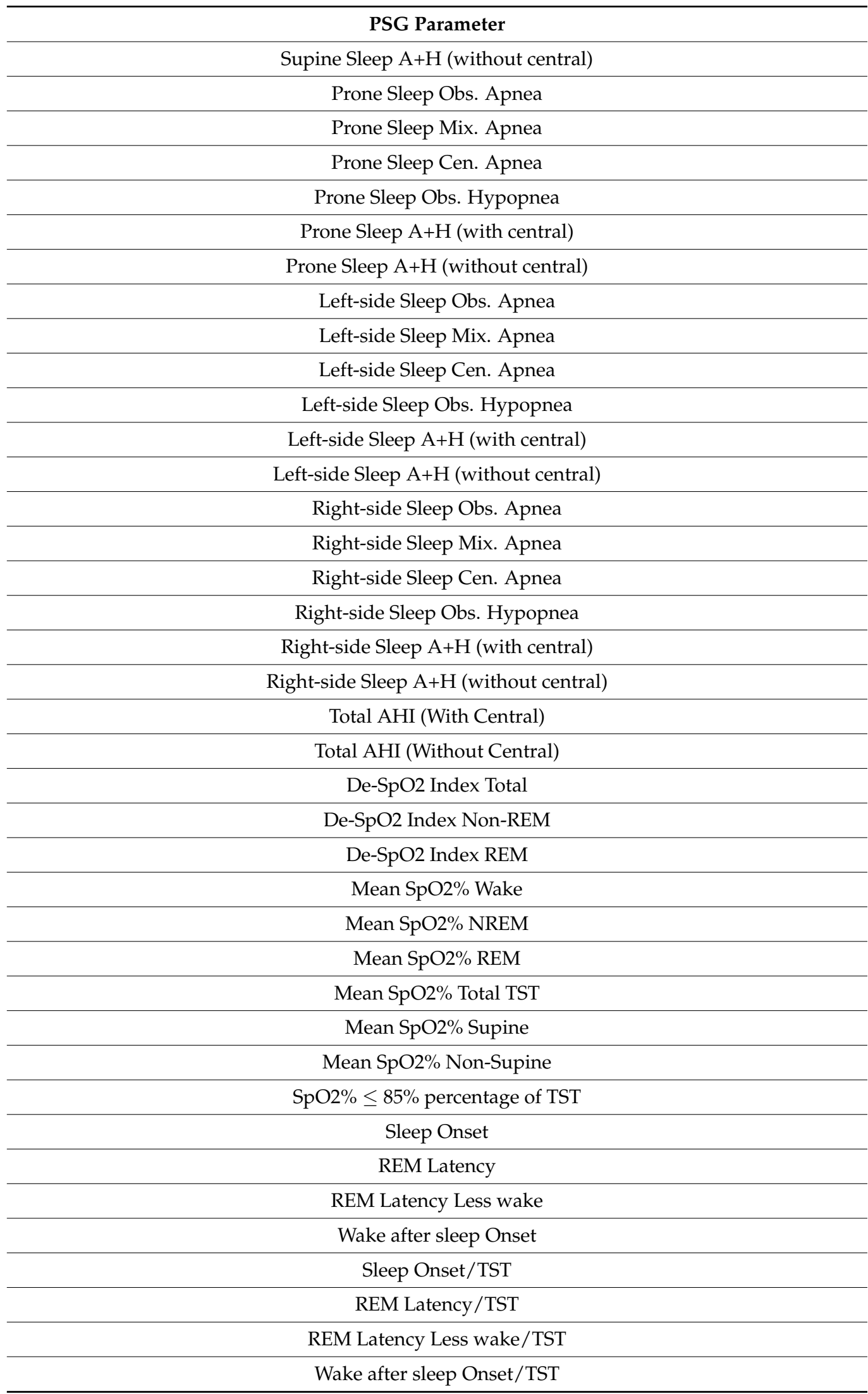

Table A2 shows the best classification results for each PSG parameter. Furthermore, it shows the validation and blind testing classification accuracies for one of the data configurations. 
Table A2. The validation accuracy for classifying data around the selected threshold using the outcomes of the bilinear models for each of the three severity groups separately.

\begin{tabular}{|c|c|c|c|c|c|c|c|}
\hline PSG Parameters & Threshold & $\mathrm{VCA}_{2}$ Total & $\mathrm{VCA}_{2}<15$ & $\mathrm{VCA}_{2 \_}>15$ & TCA $_{2}$ Total & $\mathbf{T C A}_{2}<15$ & $\mathrm{TCA}_{2}>15$ \\
\hline Stage 3 Duration \% & $10 \%$ & $85.71 \%$ & $85.00 \%$ & $84.44 \%$ & $75.00 \%$ & $83.33 \%$ & $25.00 \%$ \\
\hline Total Arousal Index & 25 & $70.87 \%$ & $72.88 \%$ & $63.64 \%$ & $80.95 \%$ & $85.00 \%$ & $0.00 \%$ \\
\hline Apnea Total Index & 1 & $79.46 \%$ & $75.00 \%$ & $84.09 \%$ & $82.61 \%$ & $81.82 \%$ & $100.00 \%$ \\
\hline Apnea Non-REM Index & 1 & $83.33 \%$ & $86.57 \%$ & $78.72 \%$ & $90.00 \%$ & $92.59 \%$ & $66.67 \%$ \\
\hline Hypopnea Total Index & 10 & $90.09 \%$ & $96.83 \%$ & $81.25 \%$ & $85.71 \%$ & $82.61 \%$ & $100.00 \%$ \\
\hline Hypopnea Non-REM Index & 7.5 & $81.74 \%$ & $81.82 \%$ & $75.51 \%$ & $85.71 \%$ & $80.00 \%$ & $100.00 \%$ \\
\hline Hypopnea Total Index (No Central) & 5 & $83.04 \%$ & $79.03 \%$ & $86.00 \%$ & $93.33 \%$ & $91.67 \%$ & $100.00 \%$ \\
\hline Supine Sleep Obs. Apnea & 1 & $75.24 \%$ & $70.97 \%$ & $79.07 \%$ & $86.96 \%$ & $86.36 \%$ & $100.00 \%$ \\
\hline Supine Sleep Obs. Hypopnea & 15 & $81.31 \%$ & $80.65 \%$ & $82.22 \%$ & $77.78 \%$ & $76.19 \%$ & $83.33 \%$ \\
\hline Supine Sleep A+H (with central) & 15 & $86.67 \%$ & $86.89 \%$ & $86.36 \%$ & $88.89 \%$ & $86.96 \%$ & $100.00 \%$ \\
\hline Total AHI (With Central) & 15 & $83.48 \%$ & $89.23 \%$ & $76.00 \%$ & $86.67 \%$ & $87.50 \%$ & $83.33 \%$ \\
\hline Total AHI (Without Central) & 15 & $86.84 \%$ & $93.75 \%$ & $76.00 \%$ & $85.71 \%$ & $85.71 \%$ & $85.71 \%$ \\
\hline De-SpO2 Index Total & 15 & $81.42 \%$ & $84.13 \%$ & $74.00 \%$ & $92.86 \%$ & $100.00 \%$ & $75.00 \%$ \\
\hline Mean SpO2\% Total TST & $94 \%$ & $69.09 \%$ & $63.93 \%$ & $75.51 \%$ & $72.00 \%$ & $68.42 \%$ & $83.33 \%$ \\
\hline Mean SpO2\% Supine & $94 \%$ & $81.40 \%$ & $78.00 \%$ & $80.56 \%$ & $81.25 \%$ & $91.67 \%$ & $50.00 \%$ \\
\hline Mean SpO2\% Non-Supine & $93 \%$ & $86.09 \%$ & $87.10 \%$ & $84.91 \%$ & $76.19 \%$ & $72.22 \%$ & $100.00 \%$ \\
\hline Wake after sleep Onset/TST & 0.15 & $73.53 \%$ & $67.80 \%$ & $76.74 \%$ & $69.23 \%$ & $66.67 \%$ & $50.00 \%$ \\
\hline
\end{tabular}

$\mathrm{V}$ : validation, $\mathrm{T}$ : blind testing, $\mathrm{CA}_{2}$ : classification accuracy using the outcomes of the second-order models, Total: all data, $<15$ : group of $\mathrm{AHI}<15$, and $>15$ : group of $\mathrm{AHI}>15$.

\section{References}

1. American Academy of Sleep Medicine. International Classification of Sleep Disorders: Diagnostic and Coding Manual; American Academy of Sleep Medicine: Westchester, NY, USA, 2005.

2. Benjafield, A.V.; Ayas, N.T.; Eastwood, P.R.; Heinzer, R.; Ip, M.S.; Morrell, M.J.; Nunez, C.M.; Patel, S.R.; Penzel, T.; Pépin, J. Estimation of the global prevalence and burden of obstructive sleep apnoea: A literature-based analysis. Lancet Respir. Med. 2019, 7, 687-698. [CrossRef]

3. Young, T.; Finn, L.; Peppard, P.E.; Szklo-Coxe, M.; Austin, D.; Nieto, F.J.; Stubbs, R.; Hla, K.M. Sleep disordered breathing and mortality: Eighteen-year follow-up of the Wisconsin sleep cohort. Sleep 2008, 31, 1071-1078.

4. Nagappa, M.; Liao, P.; Wong, J.; Auckley, D.; Ramachandran, S.K.; Memtsoudis, S.; Mokhlesi, B.; Chung, F. Validation of the STOP-Bang questionnaire as a screening tool for obstructive sleep apnea among different populations: A systematic review and meta-analysis. PLoS ONE 2015, 10, e0143697. [CrossRef] [PubMed]

5. El-Sayed, I.H. Comparison of four sleep questionnaires for screening obstructive sleep apnea. Egypt. J. Chest Dis. Tuberc. 2012, 61, 433-441. [CrossRef]

6. Gross, J.B.; Apfelbaum, J.L.; Caplan, R.A.; Connis, R.T.; Cote, C.J.; Nickinovich, D.G.; Ward, D.S.; Weaver, E.M.; Ydens, L. Practice guidelines for the perioperative management of patients with obstructive sleep apnea an updated report by the American society of anesthesiologists task force on perioperative management of patients with obstructive sleep apnea. Anesthesiology 2014, 120, 268-286.

7. Elwali, A.; Meza-Vargas, S.; Moussavi, Z. Using tracheal breathing sounds and anthropometric information for screening obstructive sleep apnoea during wakefulness. J. Med. Eng. Technol. 2019, 43, 111-123. [CrossRef]

8. Elwali, A.; Moussavi, Z. A novel Decision Making procedure during Wakefulness for Screening obstructive Sleep Apnea using Anthropometric information and tracheal Breathing Sounds. Sci. Rep. 2019, 9, 1-12. [CrossRef]

9. Elwali, A.; Moussavi, Z. Determining Breathing Sound Features Representative of Obstructive Sleep Apnea During Wakefulness with Least Sensitivity to Other Risk Factors. J. Med. Biol. Eng. 2019, 39, 230-237. [CrossRef]

10. Kapur, V.K.; Auckley, D.H.; Chowdhuri, S.; Kuhlmann, D.C.; Mehra, R.; Ramar, K.; Harrod, C.G. Clinical Practice Guideline for Diagnostic Testing for Adult Obstructive Sleep Apnea: An American Academy of Sleep Medicine Clinical Practice Guideline. J. Clin. Sleep Med. 2017, 13, 479-504. [CrossRef]

11. Epstein, L.J.; Kristo, D.; Strollo, P.J., Jr.; Friedman, N.; Malhotra, A.; Patil, S.P.; Ramar, K.; Rogers, R.; Schwab, R.J.; Weaver, E.M.; et al. Adult Obstructive Sleep Apnea Task Force of the American Academy of Sleep Medicine Clinical guideline for the evaluation, management and long-term care of obstructive sleep apnea in adults. J. Clin. Sleep Med. 2009, 5, $263-276$. 
12. Otero, A.; Félix, P.; Presedo, J.; Zamarrón, C. An evaluation of indexes as support tools in the diagnosis of sleep apnea. Ann. Biomed. Eng. 2012, 40, 1825-1834. [CrossRef] [PubMed]

13. Finkelstein, Y.; Wolf, L.; Nachmani, A.; Lipowezky, U.; Rub, M.; Berger, G. Velopharyngeal anatomy in patients with obstructive sleep apnea versus normal subjects. J. Oral Maxillofac. Surg. 2014, 72, 1350-1372. [CrossRef] [PubMed]

14. Lan, Z.; Itoi, A.; Takashima, M.; Oda, M.; Tomoda, K. Difference of pharyngeal morphology and mechanical property between OSAHS patients and normal subjects. Auris Nasus Larynx 2006, 33, 433-439. [CrossRef]

15. Elwali, A.; Moussavi, Z. Obstructive sleep apnea screening and airway structure characterization during wakefulness using tracheal breathing sounds. Ann. Biomed. Eng. 2017, 45, 839-850. [CrossRef]

16. Proakis, J. DG Monolakis-Digital Signal Processing; Prentice Hall: Upper Saddle River, NJ, USA, 2006 ; p. 911.

17. Nikias, C.L.; Raghuveer, M.R. Bispectrum estimation: A digital signal processing framework. Proc. IEEE 1987, 75, 869-891. [CrossRef]

18. Ohayon, M.M.; Carskadon, M.A.; Guilleminault, C.; Vitiello, M.V. Meta-analysis of quantitative sleep parameters from childhood to old age in healthy individuals: Developing normative sleep values across the human lifespan. Sleep 2004, 27, 1255-1273. [CrossRef] [PubMed]

19. Boselli, M.; Parrino, L.; Smerieri, A.; Terzano, M.G. Effect of age on EEG arousals in normal sleep. Sleep 1998, 21, $361-367$.

20. Majumdar, S.R.; Eurich, D.T.; Gamble, J.; Senthilselvan, A.; Marrie, T.J. Oxygen saturations less than $92 \%$ are associated with major adverse events in outpatients with pneumonia: A population-based cohort study. Clin. Infect. Dis. 2011, 52, 325-331. [CrossRef]

21. Chung, F.; Liao, P.; Elsaid, H.; Islam, S.; Shapiro, C.M.; Sun, Y. Oxygen desaturation index from nocturnal oximetry: A sensitive and specific tool to detect sleep-disordered breathing in surgical patients. Anesth. Analg. 2012, 114, 993-1000. [CrossRef]

22. Benjamini, Y.; Hochberg, Y. Controlling the false discovery rate: A practical and powerful approach to multiple testing. J. R. Stat. Soc. Ser. B Methodol. 1995, 57, 289-300. [CrossRef]

23. Chatterjee, S.; Hadi, A.S. Influential observations, high leverage points, and outliers in linear regression. Stat. Sci. 1986, 1, 379-393.

24. Spearman, C. The proof and measurement of association between two things. Int. J. Epidemiol. 2010, 39, 1137-1150. [CrossRef]

25. Elwali, A. Fast and Accurate Screening of Obstructive Sleep Apnea Disorder by Analyzing a Few Tracheal Breathing Sounds Recorded While the Individuals Are Awake. Ph.D. Thesis, University of Manitoba, Winnipeg, MB, Canada, May 2020.

26. Breiman, L. Random forests. Mach. Learn. 2001, 45, 5-32. [CrossRef]

27. Hajipour, F.; Jozani, M.J.; Elwali, A.; Moussavi, Z. Regularized logistic regression for obstructive sleep apnea screening during wakefulness using daytime tracheal breathing sounds and anthropometric information. Med. Biol. Eng. Comput. 2019, 57, 2641-2655. [CrossRef] [PubMed]

28. Petrofsky, J.S.; Laymon, M.S.; Khowailed, I.A.; Fisher, S.; Mills, A. The Effect of BMI on Oxygen Saturation at Rest and During Mild Walking. J. Appl. Med. Sci. 2015, 4, 1-8.

29. Simply, R.M.; Dafna, E.; Zigel, Y. Obstructive sleep apnea (OSA) classification using analysis of breathing sounds during speech. In Proceedings of the 2018 26th European Signal Processing Conference (EUSIPCO), Rome, Italy, 3-7 September 2018; pp. 1132-1136.

30. Wu, Y.; Liu, J.; He, B.; Zhang, X.; Yu, L. Adaptive Filtering Improved Apnea Detection Performance Using Tracheal Sounds in Noisy Environment: A Simulation Study. BioMed Res. Int. 2020, 2020, 7429345. [CrossRef]

31. Solà-Soler, J.; Fiz, J.A.; Torres, A.; Jané, R. Identification of obstructive sleep apnea patients from tracheal breath sound analysis during wakefulness in polysomnographic studies. In Proceedings of the 36th Annual International Conference of the IEEE Engineering in Medicine and Biology Society (EMBC), Chicago, IL, USA, 26-30 August 2014; pp. 4232-4235. 